\title{
B-physics overview in ATLAS
}

\author{
F. Derue D* $^{\mathrm{a} *}$
}

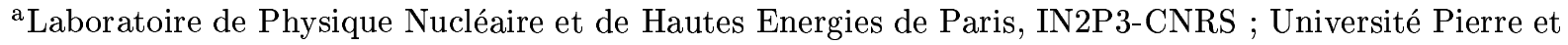
Marie Curie-Paris6 ; Université Denis Diderot-Paris7, 4 place Jussieu, tour $33 \mathrm{RdC}, 75252$ Paris Cedex 5, France

An overview of the ATLAS B-physics trigger and offline performance studies is presented. From the initial running at low luminosity at LHC, high-statistics B analyzes will allow sensitive tests of possible new physics contributions by searching for additional CP violating effects and for anomalous rates of rare B-decay channels. In the physics of the $B_{s}^{0}$ meson system the expected ATLAS sensitivities to mass and width differences are compared to recent measurements done at the Tevatron. There is also sensitivity to a weak mixing phase beyond the Standard Model expectation. ATLAS will also be able to continue its search for the rare B decays using the high luminosity running.

\section{The ATLAS B-physics potential and the trigger strategy}

ATLAS is a general-purpose detector that will run at the Large Hadron Collider (LHC) at CERN. It is designed with both discovery physics and high precision measurements. The most important subsystem in ATLAS for B-physics is the inner detector, consisting of three pixel layers, micro-strip layers and wheels and a transition radiation tracker (TRT), all immersed in a $2 \mathrm{~T}$ solenoidal magnetic field. Electron identification is performed by the TRT and the liquid argon electromagnetic calorimeter. Hadronic calorimetry is provided by the tile calorimeter and by a liquid argon calorimeter. Muon identification is performed mostly by the muon spectrometer.

The study of B-physics gives an opportunity to check the Standard Model predictions in a high perturbative order, to search for new physics, to constrain the CKM matrix elements and to provide new information on long-distance QCD effects in matrix elements of the tensor currents.

The expected fraction of events in ATLAS containing a $b \bar{b}$ pair is expected to be about $1 \%$. The LHC luminosity and energies will rise in different stages - from an energy of $900 \mathrm{GeV}$ in the center of mass and a luminosity of $10^{31} \mathrm{~cm}^{-2} \mathrm{~s}^{-1}$ in 2007 , through the nominal $14 \mathrm{TeV}$ energy and low lu-

\footnotetext{
*on behalf of the ATLAS B-physics working group
}

minosity period in $2008\left(2 \times 10^{33} \mathrm{~cm}^{-2} \mathrm{~s}^{-1}\right)$, and up to high luminosity period $\left(10^{34} \mathrm{~cm}^{-2} \mathrm{~s}^{-1}\right)$ after 2010.

At low luminosity $b \bar{b}$ pairs will be produced at a rate of about $10^{6} \mathrm{~Hz}$, but only approximately $10 \mathrm{~Hz}$ can be written to mass storage for B-physics programme. A three stage trigger system performs this reduction, retaining the events of most interest. A flexible trigger strategy [1] has been developed based on a di-muon trigger. The additional trigger menus for B-physics will require a jet or an electromagnetic cluster to be identified in addition to a single muon by the first level trigger. All selected objects guide the reconstruction at level-2 and the result will seed the reconstruction in the Event Filter. These triggered $b \bar{b}$ pairs are typically produced at medium transverse momentum in the central rapidity region.

In comparison with B-factories the LHC can study the rare $B_{s}^{0}$ decays $\left(B_{s}^{0} \rightarrow \phi \gamma, B_{s}^{0} \rightarrow\right.$ $\left.\phi \mu^{+} \mu^{-} \ldots\right)$ and $\Lambda_{b}$ decays $\left(\Lambda_{b} \rightarrow \Lambda J / \psi, \Lambda_{b} \rightarrow\right.$ $\left.\Lambda \mu^{+} \mu^{-} \ldots\right)$, rare decays of $B_{d, s}^{0}$ mesons with extremely small branching ratios $\left(\leq 10^{-9}\right)$ such as $B_{d, s}^{0} \rightarrow \gamma \mu^{+} \mu^{-}$and $B_{d, s}^{0} \rightarrow \mu^{+} \mu^{-}$, differential distributions for rare semi-leptonic $B_{d, s}^{0}$-meson decays with high accuracy. It should be pointed out that these distributions are very sensitive to Standard Model extensions. As compared with Tevatron, the LHC can produce fifty times more $b \bar{b}$ pairs. 


\section{Data samples}

Series of Monte Carlo data production, called Data Challenge (DC), has started in 2002. Their aim is the validation of the computing and software models. They also provide large data samples for the preparation of physics analyzes. All B-physics studies presented in this article are estimated by generating the physics events (in general Pythia as Monte Carlo generator), the response of the detector through a Geant simulation, and applying the online and offline reconstruction algorithms. The final analyzes are performed on the reconstructed objects. It should be noted that since the two last DCs, the production is done under the computing grid. It is an important achievement as all the LHC computing will be done under the grid[2].

In order to test the physics performance of the proposed detector, several series modules were tested in the $\mathrm{H} 6$ and $\mathrm{H} 8$ beam lines at the CERN SPS accelerator over the past years. In the year 2004, ATLAS has been involved in a huge combined test beam effort in the $\mathrm{H} 8$ beam line. A full slice of the ATLAS experiment has been tested with beams of different particles (pions, electrons, protons, muons and photons) at different energies and polarities, ranging from 1 up to $350 \mathrm{GeV}$. It has provided a unique opportunity to evaluate the individual sub-detector performances, but also to exploit their full power for detailed particle identification and measurements. It also helped to understand better the detector before the commissioning phase. These data will be used in the following to illustrate some detector performance needed for the B-physics programme and already achieved in these test beams.

\section{CP violation studies}

The full proper-time and angular analyzes allow the investigation of several parameters of physics interest using the $B_{s}^{0} \rightarrow J / \psi \phi$ decays. If the Standard Model expectations are correct, the weak phase $\left(\phi_{s}\right)$ will not be measured with useful significance, but the measurements are sensitive to the larger values predicted in some nonstandard models. The sensitivity will improve if the analyzes are combined with $B_{s}^{0}$ mixing observables in a simultaneous fit.

In ATLAS $\Delta m_{s}$ measurement can be achieved at low luminosity, already with an integral of $\sim 10 \mathrm{fb}^{-1}$.

\subsection{Tagging}

Many studies require that the produced flavour of the B-hadron (i.e if it was a particle or an antiparticle) is known. The tagging methods come in two forms. From same-side tagging, the information contained in the fragmentation tracks in the jet containing the reconstructed B-hadron is used to determine the original flavour; in ATLAS a momentum-weighted charge sum or "jetcharge" is constructed of the charge of soft decay hadrons accompanying the reconstructed $B$. For other-side tagging, a semileptonic decay lepton is sought in the other b-jet, which reveals the flavour of both jets by inference. Mistagging introduces a dilution in the observed asymmetries; the dilution is $1-2 w_{\text {tag }}$; where $w_{\text {tag }}$ is the wrong-tag fraction. The tag leads to an inefficiency. Typically, lepton tags give a low efficiency and dilution. In case the lepton tag is an electron the efficiency is 0.012 and the dilution is 0.27 . The electron tagging need the identification of electrons in jets which is done by a dedicated algorithm. This algorithm has already been efficiently tested during the 2004 combined test beam. More details can be found in [3] on the reconstruction of electrons with fully simulated data as well as real data. In case the tag lepton is a muon (so that there are three muons in the event), the Level 1 trigger muon could be either one of the $J / \psi$ legs or that muon, leading to a higher tagging efficiency of 0.025 , while keeping a dilution of 0.24 . Hadronic tags give high efficiency but large dilution (resp. 0.63 and 0.38 ).

\subsection{Precise $\sin (2 \beta)$ measurement with $B_{d}^{0} \rightarrow J / \psi K_{s}$}

ATLAS sensitivity to the $\sin (2 \beta)$ measurement with the $B_{d}^{0} \rightarrow J / \psi K_{s}$ decay was estimated on Monte Carlo fully simulated date with a maximum likelihood method.

ATLAS will achieve high sensitivity in this channel with dedicated triggers selecting 
Table 1

$B_{d}^{0} \rightarrow J / \psi K_{s}$ - summary of the expected event yields after $30 \mathrm{fb}^{-1}$ at low luminosity and the results obtained with different tagging methods and trigger selection. The trigger transverse momentum thresholds in $\mathrm{GeV}$ are indicated in brackets.

\begin{tabular}{lccc}
\hline & $J / \psi(\mu 6 \mu 5)$ & $J / \psi(\mu 6 \mu 3)$ & $J / \psi(e 1 e 1)$ \\
\hline Number of reconstructed events & $490 \mathrm{k}$ & $250 \mathrm{k}$ & $15 \mathrm{k}$ \\
Signal/background & 28 & 32 & 16 \\
$\delta \sin (2 \beta)_{\text {stat }}$ lepton tag & 0.023 & 0.030 & 0.018 \\
$\delta \sin (2 \beta)_{\text {stat }}$ jet-charge tag & 0.015 & 0.019 & - \\
$\delta \sin (2 \beta)_{\text {stat }}$ combined tag & 0.0126 & 0.016 & 0.018 \\
\hline$\delta \sin (2 \beta)_{\text {stat }} J / \psi(\mu 6 \mu 3)+J / \psi(e 1 e 1)$ & & & 0.010 \\
$\delta \sin (2 \beta)_{\text {stat }} J / \psi(\mu 6 \mu 5)$ & & 0.016 \\
\hline
\end{tabular}

$J / \psi \rightarrow \mu^{+} \mu^{-}\left(e^{+} e^{-}\right)$and by several tagging methods as described above. The expected event yields and the precision obtained with different tagging methods are summarized in Tab. 1. Results are given for several trigger options. Maximal performance can be achieved combining the lower-threshold dimuon trigger $\mu 6 \mu 3$ (the numbers correspond to the $p_{T}$ threshold of the selected leptons) with the very low threshold trigger selecting $\mu 6$ at level 1 and a pair of electrons $(e 1 e 1)$ by a global track search at level 2. Combining all tags, a precision on $\sin (2 \beta)$ of 0.01 could be achieved with $30 \mathrm{fb}^{-1}$ at low luminosity. It was estimated that the corresponding systematic uncertainty was 0.005 .

\section{3. $\Delta m_{s}$ measurement}

In $B_{s}^{0} \rightarrow D_{s} \pi, B_{s}^{0} \rightarrow D_{s} a_{1}$, the probability $p_{-}$that an initially (time $\mathrm{t}=0$ ) pure $B_{s}^{0}$ will be observed as $\bar{B}_{s}^{0}$, and the probability $p_{+}$that it will remain a $B_{s}^{0}$ are described in terms of $\Gamma, \Delta \Gamma_{s}$ and $\Delta m_{s}$ by following formula :

$$
p_{ \pm}(t)=e^{-\Gamma t}\left(\cosh \frac{\Delta \Gamma_{s}}{2} t \pm \cos \Delta m_{s} t\right) \frac{\Gamma^{2}-\Delta \Gamma_{s}^{2}}{2 \Gamma}
$$

The parameter $\Delta m_{s}$ can be derived from the ratio :

$$
r(t)=\frac{p_{+}(t)-p_{-}(t)}{p_{+}(t)+p_{-}(t)}=\frac{\cos \Delta m_{s} t}{\cosh \frac{\Delta \Gamma_{s}}{2} t}
$$

which is diluted in the case of $\Delta \Gamma_{s} \neq 0$ by the time dependent hyperbolic function. It was

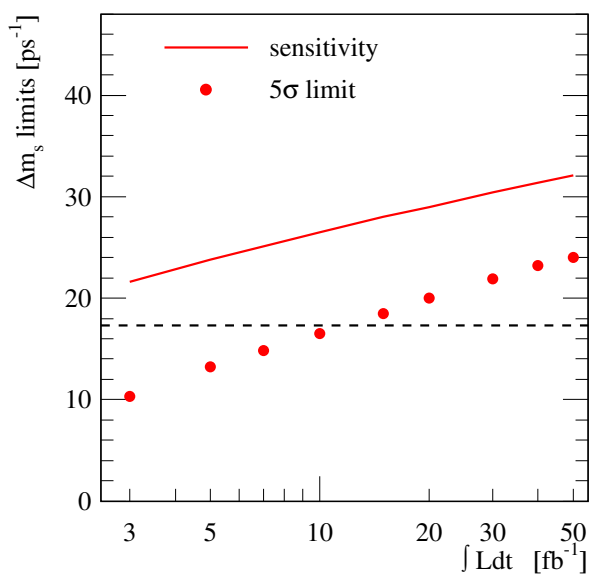

Figure 1. The dependence of $\Delta m_{s}$ measurement limits with the integrated luminosity. Dots correspond to the obtained ATLAS sigma limit, the full line correspond to the sensitivity, the dashed line to the CDF measurement.

shown however that for $\Delta \Gamma_{s} / \Gamma \leq 0.2$ no significant change in the sensitivity range of $\Delta m_{s}$ is expected. The ATLAS performance parameters for $B_{s}^{0} \rightarrow D_{s} \pi$ and $B_{s}^{0} \rightarrow D_{s} a_{1}$ processes and the background were determined by detector simulation and used as input to a fit in repeated Monte 
Carlo experiments. As regard to the $\Delta m_{s}$ measurement done by the CDF collaboration [4], a $5 \sigma$ limit could be obtained already with $\sim 10 \mathrm{fb}^{-1}$ at low luminosity (cf. Fig 1 and [5]).

\subsection{Weak phase $\phi_{s}$ in $B_{s}^{0} \rightarrow J / \psi \phi$}

The $B_{s}^{0} \rightarrow J / \psi \phi$ decay leads to three final state helicity configurations and their linear combinations are $\mathrm{CP}$ eigenstates with different $\mathrm{CP}$ parities. This means that it is not possible to extract a CP-violating weak mixing phase $\phi_{s}=$ $\arg \left(V_{c s}^{*} V_{c b} / V_{c s} V_{c b}^{*}\right)$ if the helicity amplitudes are not separated. The experimental observables are three independent angles and the $B_{s}^{0}$ proper time of the decay $B_{s}^{0} \rightarrow J / \psi \phi \rightarrow \mu^{+} \mu^{-} K^{+} K^{-}$. In large part of the events the inital $B_{s}^{0}$ flavour can also be tagged. A determination of the background contribution and its physics characteristics brings other important information. The ATLAS precision for these measurements was determined with simulated data and was used as input to angular analyzes based on a maximum likelihood fit in repeated Monte Carlo experiments. The difference of the mass eigenstate decay rates, $\Delta \Gamma_{s}$, their average value $\Gamma_{s}$ and the weak phase $\phi_{s}$ were simultaneously determined along with the two helicity amplitude values and their strong phases. The mixing parameter $x_{s} \equiv \Delta m_{s} / \Gamma_{s}$ was assumed to be measured in $B_{s}^{0} \rightarrow D_{s} \pi$ and $B_{s}^{0} \rightarrow D_{s} a_{1}$ events and was fixed. While all eight parameters are independent in the theoretical models, the experimental resolution causes some to become correlated.

The dimuon trigger was assumed to select the events with transverse momentum larger than 6 $\mathrm{GeV}$ for the first muon and $3 \mathrm{GeV}$ for the second muon. With $30 \mathrm{fb}^{-1}$ at low luminosity, the value of $\Delta \Gamma_{s}$ can be determined with a relative error of $12 \%$. The precision of the $\phi_{s}$ determination depends on the value of $x_{s}$ and on the propertime resolution. The ATLAS discovery line in the $\left(\Delta m_{s}-\phi_{s}\right)$ plane is displayed in Fig. 2, together with regions allowed by the Standard Model and by an example of new physics model. The ATLAS precision is high enough to be sensitive to new physics.

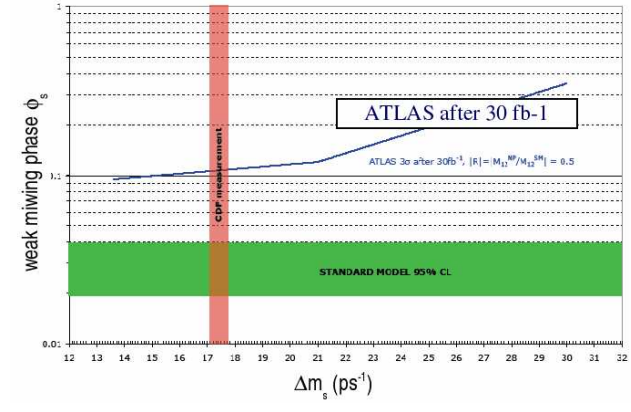

Figure 2. The $\left(\Delta m_{s}-\phi_{s}\right)$ region allowed by the Standard Model (horizontal band), the CDF measurement (vertical band) and the region of experimental sensitivity of ATLAS with $30 \mathrm{fb}^{-1}$ at low luminosity (full line).

\section{Rare decays prospects}

Flavour changing neutral current decays $b \rightarrow s, d$, which occur only at loop level in the Standard Model, have small exclusive branching ratios $\left(<10^{-5}\right)$. These decays are sensitive to the CKM matrix elements $\left|V_{t d}\right|,\left|V_{t s}\right|$. They are also sensitive to new physics.

In the era before LHC, some rare decays are accessible at $e^{+} e^{-}$factories and the Tevatron. In particular for $B_{d}^{0} \rightarrow K^{*} \gamma$ at time of LHC quite accurate measurements should be available. The process $B_{d}^{0} \rightarrow K^{*} \mu^{+} \mu^{-}$can be seen, however the mass and angular distributions can only be studied at LHC. Purely muonic rare decays can be observed before LHC only if they are drastically enhanced comparing to the Standard Model predictions $B r\left(B_{s}^{0} \rightarrow \mu^{+} \mu^{-}\right)=(3.5 \pm 1.0) \times 10^{-9}$, and $B r\left(B_{d}^{0} \rightarrow \mu^{+} \mu^{-}\right)=(1.5 \pm 1.0) \times 10^{-10}$. More details of the following sections can be found in [6].

\subsection{Purely muonic decays}

$B_{d, s}^{0} \rightarrow \mu^{+} \mu^{-}$decays have very small branching ratios in the Standard Model while they might give clear signature (due to dimuon trigger) and good sensitivity to Supersymmetry and other pos- 
sible Standard Model extensions. With an integral of $\sim 30 \mathrm{fb}^{-1}$ of the LHC operation at low luminosity, $B_{s}^{0} \rightarrow \mu^{+} \mu^{-}$decay could be detected with $2.8 \sigma$ significance (21 signal events for less than 60 for background). Adding one year with high luminosity an upper limit on the branching ration of $B_{d}^{0} \rightarrow \mu^{+} \mu^{-}$decay is set to $3 \times 10^{-10}$ with $95 \% \mathrm{CL}$, and a detection of $B_{s}^{0} \rightarrow \mu^{+} \mu^{-}$ with $4.3 \sigma$ significance (92 signal events for 660 background events).

\subsection{Semi muonic decays}

For $\Lambda_{b} \rightarrow \Lambda \mu^{+} \mu^{-}$decay, the forward-backward asymmetry $A_{F B}$ is very sensitive to Supersymmetry both for small and large values of $\check{s}$ where $\check{s}=\left(p_{\mu^{+}}+p_{\mu^{-}}\right) / M_{\Lambda_{b}}^{2} \equiv q^{2} / M_{\Lambda_{b}}^{2}$. ATLAS simulation shows that the muon triggers are not expected to change the shape of $A_{F B}$ distribution. For $30 \mathrm{fb}^{-1}$ about 800 signal events are expected. The $\check{s}$ parameter distribution is divided in three region intervals : the first from $\left(2 m_{\mu} / M_{\Lambda_{b}}\right)^{2}$ to the so-called zero-point; the second from the zeropoint to lower boundaries of $J / \psi$ and $\psi^{\prime}$ resonances and the last interval from the resonance area to $\left(M_{\Lambda_{b}}-M_{\Lambda}\right)^{2} / M_{\Lambda_{b}}^{2}$ limit. The results are shown on top of Fig. 3. The three dots with error bars correspond to simulated data. Upper points set corresponds to the theoretical Standard Model predictions and the lower set corresponds to one of possible non-standard model prediction for $C_{7 \gamma}^{S M} / C_{7 \gamma}^{e f f}<0, C_{i \gamma}^{\alpha}$ being the Wilson coefficients. ATLAS will be able to separate Standard Model from some of its extensions with high confidence level.

The branching ratios for the semi-leptonic decays $B_{d}^{0} \rightarrow K^{*} \mu^{+} \mu^{-}$and $B_{s}^{0} \rightarrow \phi \mu^{+} \mu^{-}$are rather sensitive to the Standard Model extensions, but the differential distributions, especially for $A_{F B}$ are very sensitive to Supersymmetry extension. Full ATLAS detector simulation and reconstruction of rare semi-muonic $B_{d}^{0}$ decay was performed with low luminosity. During modeling, the theoretical-based matrix element was used. A total of 2500 signal and less than 10000 background events are expected for $30 \mathrm{fb}^{-1}$ for $B_{d}^{0} \rightarrow$ $K^{*} \mu^{+} \mu^{-}$and 900 signal and less than 10000 background events for $B_{s}^{0} \rightarrow \phi \mu^{+} \mu^{-}$. In Fig. 3 (bottom) the asymmetry is presented for $B_{d}^{0} \rightarrow$

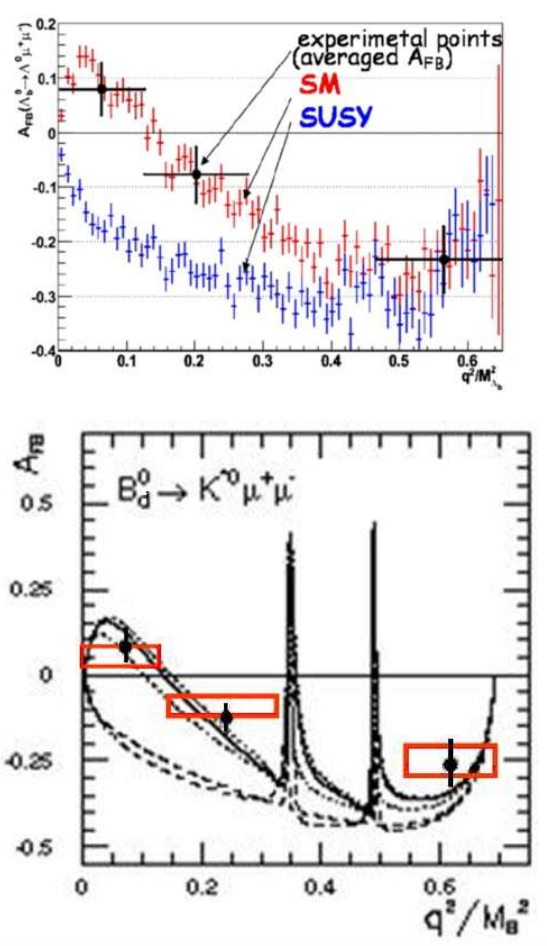

Figure 3. (Top) Forward-backward asymmetry for $\Lambda_{b} \rightarrow \Lambda \mu^{+} \mu^{-}$decay. (Bottom) Forwardbackward asymmetry for $B_{d}^{0} \rightarrow K^{*} \mu^{+} \mu^{-}$decay. See text for more details.

$K^{*} \mu^{+} \mu^{-}$decay. Solid line corresponds to Standard Model predictions according to. Dashed and dotted lines represent boundaries for MSSM predictions. The three bold dots with error bars correspond to the simulated values of $A_{F B}$. Each dot lies in one of three kinematic intervals, as for the $\Lambda_{b}$ case. It is shown that the statistics is enough, with $30 \mathrm{fb}^{-1}$ at low luminosity, for Standard confirmation and setting strong limits on Standard Model extension.

In spite of CLE0, BaBar abd Bell results it will be interesting to measure radiative penguin de- 
cay in ATLAS in $B_{d}^{0} \rightarrow K^{*} \gamma$ and $B_{s}^{0} \rightarrow \phi \gamma$. Both these decays can provide information about CKM matrix element $\left|V_{t s}\right|$ and the value of photon penguin contribution. This contribution is important for the extraction of the physics beyond Standard Model from the rare semi-leptonic B-meson decays. A simulation of these decays was performed. After $30 \mathrm{fb}^{-1}$ at low luminosity, a significance of the $B_{d}^{0} \rightarrow K^{*} \gamma$ signal will be beyond $5 \sigma$. For $B_{s}^{0} \rightarrow \phi \gamma$, the significance will be beyond $7 \sigma$. An important point in this analysis concerns the rejection of the background from $B_{d}^{0} \rightarrow K^{*} \pi^{0}$ events. A good $\pi^{0}$ rejection is needed while maintaining a high photon identification efficiency. Such a requirement also exists in other physics channels (such as $H \rightarrow \gamma \gamma$ ). This performance is made possible thanks to the fine granularity in pseudorapidity of the electromagnetic calorimeter. It has bee extensively tested with fully simulated data as well as with real data from tests beam and more details are given in [3].

\section{5. $\Lambda_{b}$ polarisation}

The study of beauty hadrons can provide important information both on te heavy quark production mechanism, leading to the polarisation of the baryon, and the dynamics of the decay. At the LHC a large number of $\Lambda_{b}$ will be produced in high energy hadronic collisions. In this environment the decay modes leading to a muon pair, which can be used for the trigger, are favourable. For this reason ATLAS has explored the primary technical challenges in the measurement of $\Lambda_{b}$ polarisation studying the decay chain $\Lambda_{b} \rightarrow \Lambda J / \psi$ with $J / \psi \rightarrow \mu \mu$ and $\Lambda \rightarrow \pi p$. An early estimation shows that for this channel it will be possible to accumulate event samples of over $60000 \Lambda_{b}$ and $\bar{\Lambda}_{b}$ (cf. Fig. 4) with the integrated luminosity of $30 \mathrm{fb}^{-1}$ at low luminosity. With a sample of this size a polarisation measurement with an accuracy of few percent should be within reach (see [7] for more details).

\section{Conclusion}

ATLAS strategy for B-physics is to concentrate efforts on the measurements that can show New

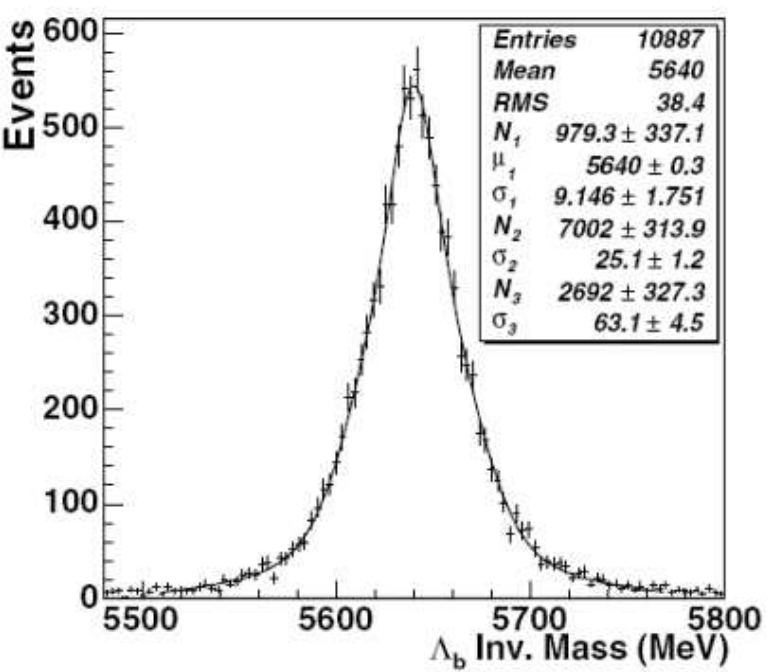

Figure 4. $\Lambda_{b}$ invariant mass distribution fitted with a triple Gaussian fit.

Physics effects or constrain New Physics models, measurements where $\mathrm{CP}$ violation effects are predicted to be small in the Standard Model, measurements where ATLAS can make a significant contribution. ATLAS proved to be capable of extracting signals of all main rare B-decays of interest at LHC.

\section{REFERENCES}

1. ATLAS HLT Technical Design Report, CERN-LHCC-2003-022

2. ATLAS Computing Technical Design Report, CERN-LHCC-2005-022

3. F. Derue (ATLAS Coll.) ATL-PHYS-CONF2005-015

4. CDF Coll., Phys. Rev. Lett. 97 (2006) 062003

5. B. Epp et al., SN-ATLAS-2002-015

6. N. Nikitin et al. (ATLAS Coll.), Nucl. Physics B (Proc. Suppl.) 156 (2006) 119-123

7. M. Biglietti et al., Nucl. Physics B (Proc. Suppl.) 156 (2006) 151-154 